\title{
ESTUDO DAS FUNCIONALIDADES DOS DRONES NA AGRICULTURA
}

\author{
STUDY OF DRONES FUNCTIONALITIES IN AGRICULTURE
}

Vinícius Parlangeli Gonçalves

Faculdade de Tecnologia de Taquaritinga - Taquaritinga - São Paulo - Brasil

Fábio Alexandre Cavichioli

Faculdade de Tecnologia de Taquaritinga - Taquaritinga - São Paulo - Brasil

DOI: 10.31510/infa.v18i1.1126

Data de submissão: 16/04/2021

Data do aceite: 09/07/2021

Data da publicação: 30/07/2021

\begin{abstract}
RESUMO
A modernização na agricultura vem se tornando algo essencial em qualquer tipo de cultivo, aliando praticidade com aumento de qualidade e de produção. Várias práticas e instrumentos tecnológicos estão sendo adotados pelos produtores para auxiliá-los, permitindo maior controle perante a cultura. Uma dessas tecnologias utilizadas cotidianamente no campo são os drones. Tratam-se de Veículos Aéreos Não Tripulados (VANT), que apresentam uma infinidade de funções, como pulverização, fotografar a plantação, identificação de possíveis danos, entre outros. $\mathrm{O}$ artigo tem como objetivo expor as funcionalidades dos drones na agricultura, buscando informações em artigos e sites com relevância. Concluísse que os drones são atualmente uma ferramenta indispensável na agricultura com potencial de crescer ainda mais e estar presente na maioria das plantações existentes.
\end{abstract}

Palavras chaves: VANT'S. Agricultura de precisão. Agricultura 4.0. Tecnologia.

\begin{abstract}
Modernization in agriculture has become something essential in any type of cultivation, combining practicality with increased quality and production. Various technological practices and instruments have been adopted by producers to assist them, allowing greater control over the culture. One of these technologies used daily in the field are drones. These are Unmanned Aerial Vehicles (UAVs), which have an infinity of functions in the area, such as spraying, photographing the plantation, identifying possible damage, among others. The article aims to expose the functionalities of drones in agriculture, seeking information in articles and websites with relevance. Cloncluding that drones are currently an indispensable tool in agriculture with the potential to grow even more and be present in most of the existing plantations.
\end{abstract}

Keywords: UAV'S. Precision agriculture. Agriculture 4.0. Technology. 


\section{INTRODUÇÃO}

Aproximando-se de seu fim, a Segunda Guerra Mundial apresentou ao mundo uma nova tecnologia, desenvolvida pelos alemães em 1944 com o intuito de usar-lhe como uma arma de detonação remota. "Buzz Boomb”, como ficou conhecido o primeiro drone, era relativamente simples, tornando-o fácil para interceptações por seguir apenas em linha reta com velocidade constante. Tinha como objetivo permitir que os soldados vigiassem ou até mesmo atacassem o território inimigo, enviar mensagens, com o intuito de não expor nenhum dos soldados. "Buzz Boomb" teve seus êxitos, levando em conta sua simplicidade. (ITARC, 2018)

Com o decorrer dos anos, avanços da tecnologia, os drones foram se aperfeiçoando, ficando menores, cada vez mais remotos, fáceis de serem transportados e ganhando novos papéis na sociedade. Papéis esses que se tornaram fundamentais em vários setores, desde filmagens de eventos até entregas de produtos. (ITARC, 2018).

Os drones que conhecemos atualmente começaram a ser criados em 1977, pelo engenheiro israelita Abe Karem, em sua viagem aos Estados Unidos. Abe, fundou sua empresa chamada "Leading System", que na época não possuía muita tecnologia. Os drones eram compostos basicamente de fibra de vidro caseiro, sobras de madeiras e motores de karts de corrida. (ITARC, 2018).

Um dos setores em que os drones ganharam muito destaque e se tornaram essenciais, foi na agricultura, uma vez que se tornaram uma das principais ferramentas para manter o controle da plantação, monitorar propriedades, entre outros.

$\mathrm{O}$ artigo tem como objetivo apresentar as funcionalidades dos drones na agricultura mostrando que é uma ferramenta muito útil na tomada de decisão do produtor rural.

\section{FUNDAMENTAÇÃO TEÓRICA}

Nesta seção abordamos as questões tecnológicas a respeito dos Drones, com o intuito de apresentar como essa ferramenta é útil na Agricultura. Artigos de relevância feitos por Gonzalo Prudkin, Fábio M. Breunig, Ariosto Mesquita, Jacqueline K. Holland, Doutor Bruce Erickson e David A. Widmar foram utilizados para o desenvolvimento do artigo. 


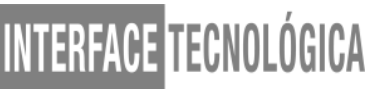

\subsection{Agricultura}

Com o passar dos anos, a agricultura brasileira atravessou diversos estágios e transformações. Nos anos 50 e 60 , menos de $2 \%$ de todas as propriedades rurais contavam com tecnologia mecânica, sendo o serviço todo realizado por humanos. (MAQCAMPO, 2019).

Com o êxodo rural em alta na época, a necessidade de mais alimentos nas cidades aumentou, fazendo com que os produtores tivessem que de alguma maneira aumentar sua produção para suprir a demanda. Com isso, foram surgindo novas tecnologias para aumentar a produtividade, como inserção das máquinas no campo, pesquisas de sementes, insumos e principalmente em implementos agrícolas e máquinas. (MAQCAMPO, 2019).

A agricultura é um aglomerado de técnicas com objetivo de cultivar terras a fim de adquirir produtos, que são os alimentos. Tendo início por volta de 11.500 anos atrás, o homem começou a cultivar as terras dando início ao nomadismo, pois não era mais necessária a busca por alimento, já que eles podiam cultivar em um lugar e se estabilizarem nesse mesmo local. Os primeiros registros de civilizações que cultivavam são das beiras dos rios Tigres, Eufrates e Nilo, na região da Mesopotâmia. (RIBEIRO, 2018).

O tempo foi passando e as tecnologias foram surgindo em todos os setores no mundo, das indústrias aos campos de plantações. Essas novas tecnologias permitiram suprir a demanda de alimentação da população e vem tornando as áreas agrícolas cada vez mais mecanizadas e automatizadas com tecnologias que vão das máquinas agrícolas, insumos, produtos químicos e até mesmo nas sementes. (RIBEIRO, 2018).

Uma nova modalidade da agricultura vem ganhando destaque nas grandes propriedades e com projeções de se tornarem cada vez mais fundamentais na agricultura de modo geral, das grandes propriedades as pequenas propriedades, se trata da Agricultura de precisão (AP). (RIBEIRO, 2018).

A agricultura de precisão é definida como um sistema para administrar áreas agrícolas baseado na variação espacial e temporal da produtividade, pretendendo aumentar a sustentabilidade e retorno econômico. (BRFÉRTIL, 2019).

\subsection{Drones}

Uma das tecnologias utilizadas na agricultura de precisão se trata dos drones ou também conhecidos como VANT'S (Veículos aéreos não tripulados). Inicialmente desenvolvidos por 


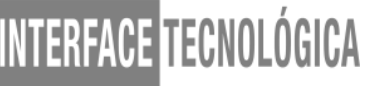

indústrias bélicas, os drones ganharam novas tarefas em outros espaços, como na agricultura. (MESQUITA, ARIOSTO, 2014).

Controlado remotamente, os drones são utilizados para realizarem vários serviços, desde os mais arriscados como espionagem por exércitos até entregas de mercadorias, filmagens e serviços variados na agricultura. (PRUDKIN et al., 2019).

\subsubsection{Tipos de drones existentes}

Os drones possuem vários modelos, desde os mais simples aos mais complexos, daqueles que executam várias funções aos que são utilizados para lazer. Os drones possuem três tipos de variedade, podendo ser: drones de asa fixa, drones multi rotores e drones de rotor único. (SENAR, 2018).

\section{Drone de rotor único}

Modelo mais simples dos drones, composto por apenas um rotor, é eficiente em vôos longevos e demoram a descarregarem. (Figura 1).

Figura 1 - Drone de rotor único.

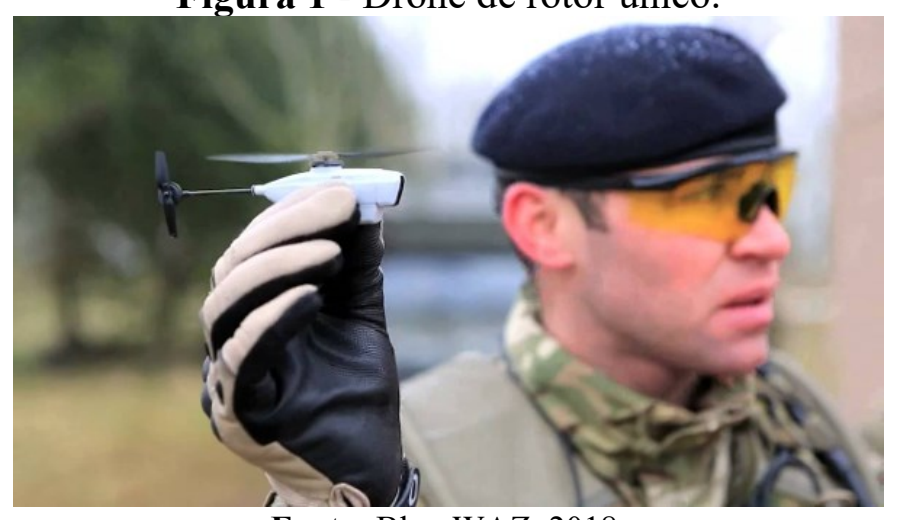

Fonte: Blog WAZ, 2018.

\section{Drone de asa fixa}

É composto por uma asa comprida, são mais utilizados em filmagens por conseguirem pairarem no ar (figura 2). 


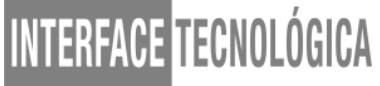

Figura 2 - Drone de asa fixa.

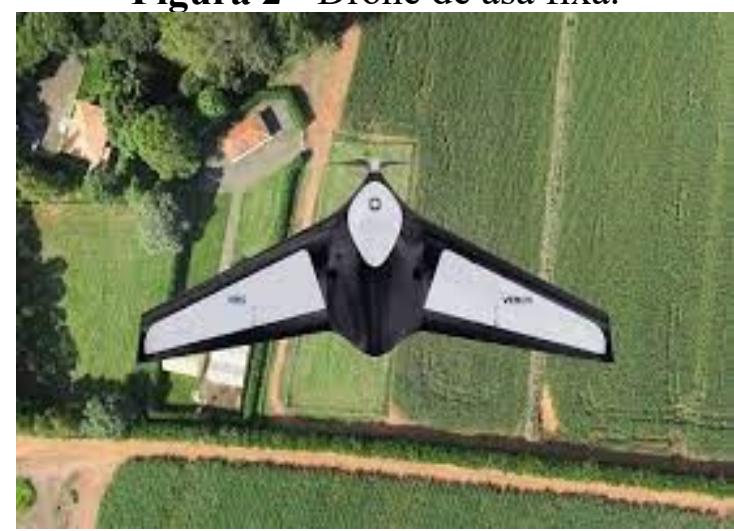

Fonte: Projeto Draft, 2018.

\section{Drones multi rotores}

Os drones multi rotores possuem mais de um rotor, podendo variar de 2 a 8 . São os mais fáceis de pilotar, já que por possuem mais de um rotor, são mais fáceis de decolar e pousar inclusive na vertical, utilizados em filmagens e inspeções, se mantém estáveis no ar. (figura 3 ).

Figura 3 - Drone com 8 rotores.

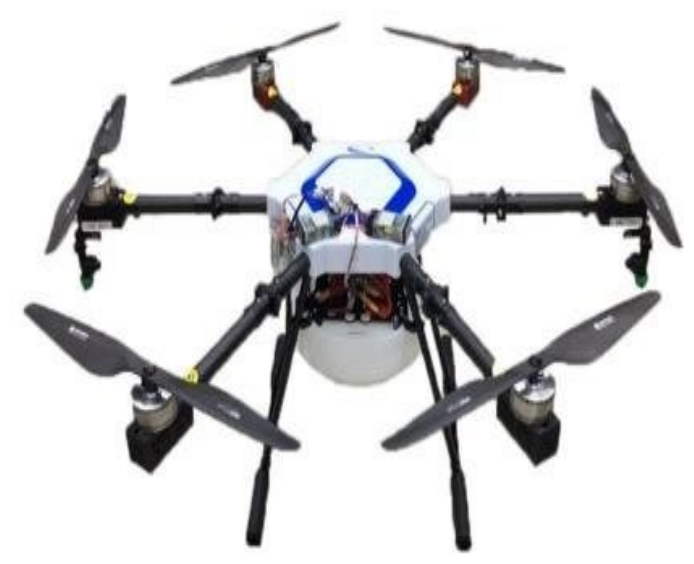

Fonte: Research Gate, 2018.

\subsection{Câmeras}

Os drones são capazes de nos mostrar imagens que dificilmente conseguiríamos ver por possuírem câmeras de alta qualidade, recolhendo imagens com alta definição e em lugares específicos e de difícil acesso. As câmeras dos drones são divididas em dois tipos, sendo câmeras RGB e NIR e câmeras multiespectrais. (DRONES E ENGENHARIA, 2020). 


\section{Câmera RGB e NIR}

Com as câmeras RGB e NIR é possível coletar dados quantitativos da área, são mais utilizadas na área de topografia, construção civil, mineração e agricultura. (DRONES E ENGENHARIA, 2020).

\section{Câmera multiespectral}

Essas câmeras possuem vários sensores, sendo cada um deles um filtro de alta qualidade, específico para captação de diversos formatos de ondas refletidas, permitindo a separação em diferentes tipos de cores, também captam frequências como o infravermelho, invisível a olho nu. (DRONES E ENGENHARIA, 2020).

\section{PROCEDIMENTOS METODOLÓGICOS}

O artigo foi desenvolvido por meio de pesquisa bibliográfica, que para Vera Regina Casari Boccato (2006), é a procura para resoluções de hipóteses por meio de artigos científicos publicados.

Com foco em obras que apontem o tema: Estudo das funcionalidades dos drones na agricultura, foram realizadas pesquisas em sites da área de agricultura de precisão, tecnologia e artigos científicos publicados. A pesquisa é de condição qualitativa, que para Lüdke e André (1986), presume o relacionamento do pesquisador com a construção do artigo.

\section{RESULTADOS E DISCUSSÕES}

Com a modernização no campo, os drones estão cada vez mais disponíveis, seja para pequenos ou grandes produtores. Segundo Bruna Eduarda Meinen Feil (2018), as vantagens do uso dos drones na agricultura são: redução do tempo de monitoramento da área cultivada, mapeamento de áreas de difícil acesso, descobrimento de adversidades na plantação (como pragas, doenças e excesso ou escassez na irrigação), medição da concentração de água e nutrientes no solo, custo reduzido e imagens de alta qualidade.

Suas desvantagens são: restrições para o voo com limite de altura, raio de operação e locais inapropriados como aeroportos, documentação necessária para pilotagem dos drones e em alguns drones multi-rotores, o baixo tempo de operação no ar. (MEINEN FEIL, 2018). 
Em poucos anos, os drones se modernizaram em toda a sua composição e com isso passaram a beneficiar setores que até então não havia muita tecnologia, como o setor da agricultura. Atualmente são mais acessíveis para os produtores, podendo adquirir um equipamento ou alugá-los, fazendo assim, com que a produtividade da plantação possa aumentar. (GIRALDELI, 2019).

De acordo com Bastos (2015), essas são as atividades que os drones desempenham no campo que merecem destaque:

A análise de cultura talvez seja o principal uso do drone na agricultura, realizam detecção de pragas ou doenças, falhas no plantio, excesso ou escassez de irrigação e análise da plantação, através de softwares que capturam imagens. (BASTOS, 2015).

O drone paira sob a cultura capturando imagens que em softwares são organizadas cronologicamente, possibilitando verificar o desenvolvimento da safra. (BASTOS, 2015).

Através de imagens capturadas pelos drones em seu vôo e utilização de softwares, possibilita se a realização de medições no campo. (BASTOS, 2015).

Com as imagens capturadas pelos drones, é possível demarcar áreas, selecionando os melhores locais para realizar o plantio e até mesmo para demarcar fontes de água e rios para preservação. (GIRALDELI, 2019). (figura 4).

Figura 4 - Demarcação de terreno realizada por drone.

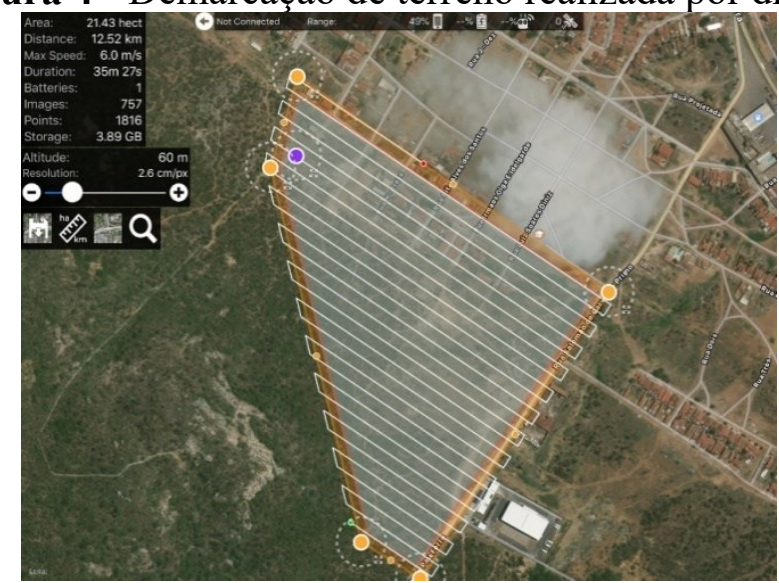

Fonte: Geosensori, 2019.

Com a alta tecnologia nos drones, a precisão deles se tornou excelente para a pulverização em lugares específicos reduzindo o custo com produtos químicos, pulverizando somente as áreas necessitadas, reduzindo em até 50\% o custo dos insumos. (GIRALDELI, 2019). 


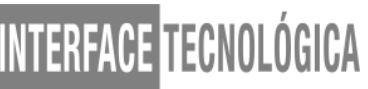

O primeiro drone a realizar testes para saber se era possível a pulverização nas plantações, foi o drone Pelicano, desenvolvido pela empresa brasileira SkyDrones. O Pelicano foi aprovado no teste e desde então começou a ser comercializado. (GIRALDELI, 2019). (figura $5)$.

Figura 5 - Drone Pelicano.

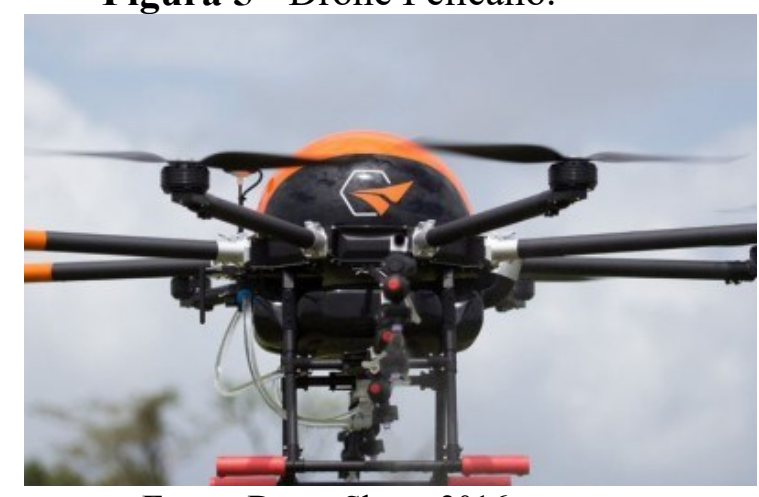

Fonte: Drone Show, 2016.

A Agência Nacional de Aviação Civil (ANAC) permite o uso de drones para pulverização desde que estes não ultrapassem os $25 \mathrm{Kg}$ (equipamento e carga), para evitar acidentes com maiores gravidades. (GIRALDELI, 2019).

Ainda de acordo com Ana Lígia Giraldeli (2019), com a utilização dos drones na agricultura, também é possível:

- Descobrir ocorrência de incêndios;

- Áreas desmatadas;

- $\quad$ Nascentes de rios;

- $\quad$ Reconhecimento de áreas com difícil acesso;

- $\quad$ Estimar produtividade;

- $\quad$ Mapeamento agrícola e hídrico;

- $\quad$ Impor a agricultura de precisão.

\section{CONSIDERAÇÕES FINAIS}

Ao passar dos anos, a agricultura foi se modernizando e ganhado novas ferramentas para auxiliarem na produção, como máquinas, implementos agrícolas, insumos e os drones.

Inicialmente utilizados em atividades bélicas, os drones entraram de vez no setor da agricultura e ganharam novas versões, cada vez mais atualizadas com capacidade de realizarem 
novas tarefas. Foram desenvolvidos novos modelos, podendo ser de rotor único, multi rotor ou de asa fixa, suas câmeras ficaram cada vez melhores, gerando imagens difíceis de enxergar a olho nu e até mesmo imagens em infravermelho.

Cada drone possui uma maneira de desempenhar uma atividade, os tempos de vôo, movimentos realizados, peso, diferenciam os três modelos existentes. Seu uso pode variar com o que é necessário, algumas dessas utilidades dos drones na agricultura, são: filmagens para monitoramento da cultura, sobrevoo em locais de difícil acesso, pulverização das plantas em lugares específicos e com precisão, delimitação de áreas, entre outros.

Com todas essas atividades desempenhadas com precisão, os drones se tornaram uma ferramenta fundamental na agricultura, com projeções de cada vez mais estar presente no campo, seja nas grandes ou pequenas propriedades, pois reduz o tempo e o custo dos produtores, por conseguinte, aumentando a produtividade.

\section{REFERÊNCIAS}

AGRICULTURA DE PRECISÃO. BRFértil, 2019. Disponível em:

https://brfertil.com.br/agricultura-de-precisao/. Acesso em: 1 de abril de 2021.

BASTOS, 2015. 15 usos de drones na agricultura e pecuária. Disponível em: https://revistagloborural.globo.com/Noticias/Pesquisa-e-Tecnologia/noticia/2015/05/15usosde-drones-na-agricultura-e-na-pecuaria.html. Acesso em: 12 de abril de 2021.

COELHO, A. M. Agricultura de precisão em sistemas agrícolas. In: FALEIRO, F.G.; FARIAS NETO, A.L. Savanas: desafios e estratégias para o equilíbrio entre sociedade, agronegócio e recursos naturais. Planaltina, DF: Embrapa Cerrados, 2008. p. 1063-1083.

COMO A AGRICULTURA SE MODERNIZOU - HISTÓRIA. MaqCampo, 2019. Disponível em: http://www.maqcampo.com.br/node/2228\#: :text=Nas\%20d\%C3\%A9cadas $\% 20 \mathrm{de} \% 2050 \% 2$ 0e,rurais $\% 20$ contavam $\% 20 \mathrm{com} \% 20 \mathrm{~m} \% \mathrm{C} 3 \% \mathrm{~A} 1$ quinas $\% 20 \mathrm{agr} \% \mathrm{C} 3 \% \mathrm{ADcolas}$.\&text $=\mathrm{Com} \%$ $20 \mathrm{o} \% 20$ passar $\% 20 \mathrm{dos} \% 20$ tempos,em $\% 20 \mathrm{~m} \% \mathrm{C} 3 \%$ A 1 quinas $\% 20 \mathrm{e} \% 20 \mathrm{implementos} \% 20 \mathrm{agr} \%$ C3\%ADcolas. Acesso em: 3 de abril de 2021.

DRONES E ENGENHARIA. TIPOS DE CÂMERAS PARA DRONES E AS IMAGENS GERADAS, 2020. Disponível em: https://blog.droneng.com.br/tipos-de-cameras-paradrones/. Acesso em: 6 de abril de 2021.

GARRET, Filipe. O que é drone e para que serve? A tecnologia invade o espaço aéreo. Tech Tudo, 2013. Disponível em: https:/www.techtudo.com.br/noticias/noticia/2013/10/o- 
que-sao-e-para-que-servem-os-drones-tecnologia-invade-o-espaco-aereo.html. Acesso em: 2 de abril de 2021.

GIRALDELI, Ana Lígia. Drones na agricultura: como eles te ajudam a lucrar mais. Aegro, 2019. Disponível em: https://blog.aegro.com.br/drones-na-agricultura/. Acesso em: 4 de março de 2021.

HOLLAND, J. K.; ERICKSON, B.; WIDMAR, D. A. Precision Agricultural services Dealership Survey Results. Sponsored by Croplife Magazine and Center for Food and Agricultural business. West Lafayette: Dept. of Agricultural Economics, Purdue University, 2013. Disponível em:https://agribusiness.purdue.edu/wp-content/uploads/2019/08/2015-croplife-purdue-precision-dealer-survey.pdf. Acesso em: 4 de abril de 2021.

ITARC. História dos drones: Como surgiram? Para que servem? 2018. Disponível em:https://itarc.org/historia-dosdrones/\#: :text=Quem $\% 20$ inventou $\% 20 \mathrm{o} \% 20 \mathrm{drone} \% 3 \mathrm{~F}$,pessoas $\% 20$ para $\% 20$ controlar $\% 20 \mathrm{u}$ m\%20drone. Acesso em: 4 de abril de 2021.

JÚNIOR DE OLIVEIRA, Altacis; FERREIRA DA SILVA, Gustavo; RODRIGUES DA SILVA, Givanildo; ALVES C. DOS SANTOS, Andressa; SOARES ALVES CALDEIRA, Daniela; KAROLINE C. VILARINHO, Marcella; ANTONIO APARECIDO BARELLI, Marco. Potencialidades da utilização de drones na agricultura de precisão. Brazilian Journal of Development, 2020.

LUDKE M., ANDRÉ M. E. D. A. Pesquisa em Educação: Abordagens Qualitativas. Temas Básicos de Educação e Ensino. São Paulo: EPU Pedagógica e Universitária Editora, 1986.

MEINEN FEIL, Bruna Eduarda. Vale a pena investir no uso dos drone na agricultura? Mais Soja. Disponível em: https://maissoja.com.br/vale-a-pena-investir-no-uso-de-drones-naagricultura/. Acesso em 10 de abril de 2021.

MESQUITA, Ariosto. O avanço dos drones. Agro DBO, 2014.

PRUDKIN, Gonzalo; M. BREUNIG, Fábio. Drones e ciência: Teoria e aplicações metodológicas. 1. ed. Santa Maria - SC: Facos UFSM, 2019.

REGINA CASARI BOCATTO, Vera. Metodologia da pesquisa bibliográfica na área odontológica e o artigo científico como forma de comunicação. Revista de odontologia da Universidade de São Paulo, 2006.

RIBEIRO, Amarolina. O que é agricultura? 2018. Brasil Escola. Disponível em: https://brasilescola.uol.com.br/o-que-e/geografia/o-que-e-agricultura.htm. Acesso em:30 de março de 2021. 
RODRIGUES NOVAES, Guilherme. Drones podem ser uma alternativa para gestão portuária? Revista eletrônica de estratégias e negócios, 2018.

SCUSSEL, Alexandre. Drones na Agricultura: prepare-se para atuar neste setor milionário. 2016. Fórum Drones, São Paulo: MundoGeo. Disponível

em:https://mundogeo.com/2016/04/15/drones-na-agricultura-prepare-se-para-atuar-nestesetor-milionario/. Acesso em: 9 de abril de 2021.

SERVIÇO NACIONAL DE APRENDIZAGEM RURAL (SENAR). Agricultura de precisão: Drones. Brasília: SENAR, 2018. 